\title{
Specificity of Human Cortical Areas for Reaches and Saccades
}

\author{
Ifat Levy, ${ }^{1}$ Denis Schluppeck, ${ }^{1}$ David J. Heeger, ${ }^{1,2}$ and Paul W. Glimcher ${ }^{1,2}$ \\ ${ }^{1}$ Center for Neural Science and ${ }^{2}$ Department of Psychology, New York University, New York, New York 10003
}

\begin{abstract}
Electrophysiological studies in monkeys have identified effector-related regions in the posterior parietal cortex (PPC). The lateral intraparietal area, for example, responds preferentially for saccades, whereas the parietal reach region responds preferentially for arm movements. However, the degree of effector selectivity actually observed is limited; each area contains neurons selective for the nonpreferred effector, and many neurons in both areas respond for both effectors. We used functional magnetic resonance imaging to assess the degree of effector preference at the population level, focusing on topographically organized regions in the human PPC [visual area V7, intraparietal sulcus 1 (IPS1), and IPS2]. An event-related design adapted from monkey experiments was used. In each trial, an effector cue preceded the appearance of a spatial target, after which a Go signal instructed subjects to produce the specified movement with the specified effector. Our results show that the degree of effector specificity is limited in many cortical areas and transitions gradually from saccade to reach preference as one moves through the hierarchy of areas in the occipital, parietal, and frontal cortices. Saccade preference was observed in visual cortex, including early areas and V7. IPS1 exhibited balanced activation to saccades and reaches, whereas IPS2 showed a weak but significant preference for reaches. In frontal cortex, areas near the central sulcus showed a clear and absolute preference for reaches, whereas the frontal eye field showed little or no effector selectivity. Although these results contradict many theoretical conclusions about effector specificity, they are compatible with the complex picture arising from electrophysiological studies and also with previous imaging studies that reported mostly overlapping saccade- and arm-related activation. The results are also compatible with theories of efficient coding in cortex.
\end{abstract}

Key words: effector specificity; eye movements; arm movements; LIP; PRR; fMRI

\section{Introduction}

The notion that movements by different effectors, such as arms and eyes, are encoded in different cortical areas is now a widely held view (Colby and Duhamel, 1991; Colby and Goldberg, 1999; Snyder et al., 2000a; Andersen and Buneo, 2002; Glimcher, 2003; Grefkes and Fink, 2005). According to this view, neurons in the lateral intraparietal area (area LIP), for example, are hypothesized to participate principally, if not exclusively, in the generation of saccadic eye movements (Gnadt and Andersen, 1988; Platt and Glimcher, 1998). In contrast, the more medial and posterior parietal reach region (PRR) has been hypothesized to represent a module dedicated primarily, again if not exclusively, to the generation of arm movements (Snyder et al., 1997; Galletti et al., 2003). This modular hypothesis is also supported by the differential corticocortical connections of areas within the posterior parietal cortex (PPC) (Asanuma et al., 1985; Blatt et al., 1990, 1996; Caminiti et al., 1996; Lewis and Van Essen, 2000) and by microstimulation studies showing that only specific regions within the PPC give rise to eye movements when stimulated (Thier and Andersen, 1996, 1998). However, both LIP and PRR

Received Nov. 22, 2006; revised March 14, 2007; accepted March 20, 2007.

This work was supported by James S. McDonnell Foundation Grant 21002079 (P.W.G.) and National Institute of Mental Health Grant R01-MH69880 (D.J.H.). We thank Joseph Kable, Brian Lau, Uri Hasson, and Margaret Grantner for fruitful discussions and comments and Edward Ryklin and the Center for Brain Imaging at New York University for technical assistance.

Correspondence should be addressed to Ifat Levy, Center for Neural Science, New York University, 4 Washington Place, Room 809, New York, NY 10003. E-mail: ifat.levy@nyu.edu.

D01:10.1523/JNEUROSCI.0459-07.2007

Copyright $\odot 2007$ Society for Neuroscience $\quad$ 0270-6474/07/274687-10\$15.00/0 have been shown to contain neurons that are either responsive to both effectors or even specific for the "wrong" effector (Snyder et al., 1997; Colby and Goldberg, 1999; Gottlieb and Goldberg, 1999). These observations challenge the hypothesis that distinct effector-specific cortical modules exist in PPC.

If effector-specific anatomic modules do exist in PPC, then functional magnetic resonance imaging (fMRI) is an ideal technique for revealing them because it reflects neural signals at the population level and hence presumably at the level of cortical modules. Several existing imaging studies have taken advantage of this property in an effort to identify the human homologs of LIP and PRR by searching for task-specific or effector-specific areas in regions such as the intraparietal sulcus (IPS). However, the results of these studies have been hard to interpret with regard to the prevailing view that effector-specific modules populate the PPC (for review, see Grefkes and Fink, 2005; Culham and Valyear, 2006) (for examples, see Kawashima et al., 1996; Connolly et al., 2000; Simon et al., 2002; Astafiev et al., 2003; Connolly et al., 2003; Medendorp et al., 2003, 2005).

We therefore set out to determine whether distinct parietooccipital areas, as defined by the topographic mapping of sensorimotor space, separately encode saccades and reaches as has been hypothesized. Previous studies (Sereno et al., 2001; Schluppeck et al., 2005, 2006; Silver et al., 2005) have shown that the PPC contains at least two regions, or modules, that exhibit a topographical organization during the planning and production of delayed saccades, as well as during orienting of covert attention. Each region has been shown to topographically map saccades into the contralateral visual field. The two areas, termed IPS1 and IPS2, are 
anterior to visual area V7 and are separated from each other and from V7 by reversals in visual field orientation. Schluppeck et al. (2005) speculated that these two areas may separately encode saccades and reaches in a manner similar to the proposed organization of LIP and the PRR in monkey posterior parietal cortex.

To test that specific hypothesis, and the more general underlying hypothesis that reaches and saccades are fully separable in parieto-occipital cortex, we measured effector preference in several other brain areas as well, ranging from V1 to frontal eye field (FEF). To accomplish this, we adapted a behavioral protocol from the monkey electrophysiological literature (Calton et al., 2002) to human fMRI.

Our results show that the transitions from encoding saccades to encoding reaches, as well as the transitions from encoding sensory-to-motor task properties, as one moves from cortical area to area, are not as distinct as might be expected from the hypothesis that effector-specific modules populate the PPC. Although there are clear transitions from saccade to reach and from sensory to motor as one moves from areas such as the primary visual cortex (V1) to areas such as primary motor cortex (M1), these transitions occur gradually across several cortical areas. Thus, although areas in the PPC exhibited preferences for one effector or the other, none showed effector specificity. In other words, although activity evoked during saccades and reaches was significantly different (with larger responses in some areas during saccades and in other areas during reaches), activity in all areas was significantly active above baseline for both movement types. These findings challenge the widely held conclusion that entirely distinct effector-specific cortical modules exist in the PPC.

\section{Materials and Methods Subjects}

Ten healthy right-handed volunteers (four women), ages $24-43$ years, participated in one or both of the experiments. Eight subjects participated in both the localizer experiment and in the main experiment, and two subjects participated in the main experiment only. A portion of the data from the localizer experiment (from three of eight subjects) has appeared previously (Schluppeck et al., 2005). Procedures were in compliance with the safety guidelines for MRI research and approved by the University Committee on Activities Involving Human Subjects at New York University. All subjects had normal or corrected-to-normal vision and provided written informed consent.

\section{Experimental protocol}

\section{MRI acquisition}

Functional magnetic resonance imaging at $3 \mathrm{~T}$ (Allegra; Siemens, Erlangen, Germany) was used to measure blood oxygen level-dependent changes in cortical activity. During each fMRI scan, a time series of volumes was acquired using a $\mathrm{T}^{*}$-weighted echo planar imaging pulse sequence. For the localizer experiment, these values were as follows: repetition time (TR), $1500 \mathrm{~ms}$; echo time (TE), $30 \mathrm{~ms}$; flip angle, $80^{\circ}$; 24 or 27 3 -mm slices with no interslice gap; in-plane resolution, $3 \times 3 \mathrm{~mm}$; field of view (FOV), $192 \mathrm{~mm}$. For the main experiment, these were as follows: TR, $1250 \mathrm{~ms}$; TE, $30 \mathrm{~ms}$; flip angle, $80^{\circ}$; 20 or 234 -mm slices with a $1 \mathrm{~mm}$ interslice gap; in-plane resolution, $3 \times 3 \mathrm{~mm}$; FOV, $192 \mathrm{~mm}$. Images were acquired using custom receptive field coils (NM-011 transmit head coil, NMSC-021 four-channel phased array receive coil, and NMSC-011 four-element parallel imaging array; NOVA Medical, Wakefield, MA). In addition, T1-weighted high-resolution $\left(1 \times 1 \times 1 \mathrm{~mm}^{3}\right)$ anatomical
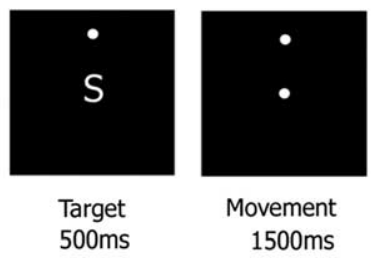

Movement

$1500 \mathrm{~ms}$
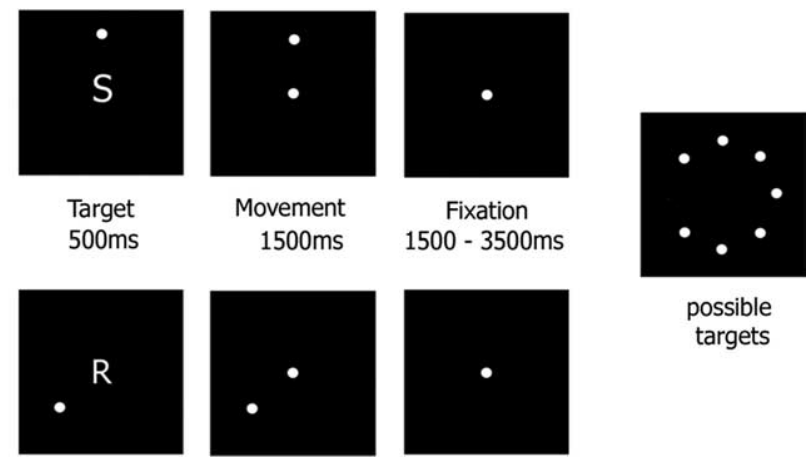

possible targets

the main experiment. Saccade and reach trials started with an effector cue ( $R$ or $S$ ), followed Figure 1. Experimental design of the main experiment. Saccade and reach trials started with an effector cue (R or $S)$, followed
by a peripheral target in one of seven possible locations. Effector cue was then replaced by a fixation point, instructing subjects to move their arm or their eyes to the target and back to central position. In neutral trials, a fixation point appeared throughout the trial, and subjects fixated and kept their index finger at the central target on the touch screen.

images were acquired with a magnetization-prepared rapid-acquisition gradient echo (MP-RAGE) pulse sequence and used for cortical segmentation, three-dimensional $(3 \mathrm{D})$ reconstruction, and volume-based statistical analysis. To minimize head movement, subjects' heads were stabilized with foam padding. Stimuli were projected onto a screen at the back of the scanner, and subjects viewed them through a mirror attached to the head coil.

\section{Localizer: topography for delayed saccades (eight subjects)}

To localize topographically organized areas in posterior parietal cortex, subjects performed a delayed saccade task in which targets appeared at successive locations "around the clock" at $11^{\circ}$ eccentricity, separated by $30^{\circ}$ angular steps (Sereno et al., 2001; Schluppeck et al., 2005). Each cycle lasted $54 \mathrm{~s}$ (number of target locations $\times$ trial duration $=12 \times 4.5 \mathrm{~s}$ ). Subjects performed the task for five cycles, resulting in scans lasting $270 \mathrm{~s}$. Scans in which the target progressed in clockwise and counterclockwise directions were interleaved.

To localize saccade-related areas in general, regardless of topography, each topography scanning session also included one or two scans (duration, $270 \mathrm{~s}$ ) in which subjects alternated between blocks of central fixation (27 s) and blocks consisting of a rapid series (one every $750 \mathrm{~ms}$ ) of eye movements ( $27 \mathrm{~s}$ ). During the eye movement blocks, subjects shifted their gaze between the central fixation spot and a series of peripheral targets. The presentation of the peripheral targets $\left(750 \mathrm{~ms}, 10^{\circ}\right.$ eccentricity, random angles) alternated with the central fixation spot $(750 \mathrm{~ms})$, only one of which was visible at any time.

\section{Main experiment: saccades and reaching (10 subjects)}

The aim of the main experiment was to look for effector selectivity for arm and eye movements. Subjects performed a series of trials making saccadic eye movements, reaching arm movements, or holding the eyes and arm stationary. The right arm was used in each scan; the upper arm rested on foam padding, and arm movements were made principally using the lower arm, with the hand closed in a fist and the index finger extended in a pointing position. Movements were made on an MRIcompatible touch screen, which could not be seen by the subject and which allowed us to monitor the movements during the experiment. The touch screen was placed on an adjustable tray attached to the scanner bed. The exact location of the touch screen and the angle respective to the subject were individually adjusted for each subject, such that arm movement was as comfortable as possible by placing the touch screen so that it lay within the workspace of both arms. This required tilting the touch screen away from the standard position (perpendicular to the torso) and toward the bed. As a result, arm movements were performed on a planar surface $\sim 45^{\circ}$ from alignment with the scanner bed and $\sim 135^{\circ}$ from alignment with the visual field (i.e., $45^{\circ}$ from the direction of gaze). Each $7.5 \mathrm{~s}$ trial began with an effector cue (reach, saccade, or neither), indicated by a letter ("R," "S," or a dot) at fixation, while subjects fixated and kept their index finger at the central target on the touch screen (Fig. 1). After a randomized delay period (2-4s), a peripheral target appeared on 
the visual display ( $2 \mathrm{~s}, 5^{\circ}$ eccentricity) at one (randomly selected) of seven possible polar angles $\left(45^{\circ}\right.$ apart, starting at $45^{\circ}$ to the left of the upper vertical meridian (UVM); the left horizontal meridian was left out because we were concerned that this target lay at the edge of the right arm workspace and would require a shoulder extension that would induce a movement artifact during our scans). The letter at fixation changed to a fixation point $500 \mathrm{~ms}$ after target appearance, cueing subjects to execute the movement specified by the target (a movement of the unseen hand or the eye having the same amplitude and direction as the fixation-to-target vector) and then to return immediately to the ocular and manual fixation. Subjects were instructed to maintain visual fixation of the central position while reaching and to keep their index finger fixed at the central target while making an eye movement. Physical distance for the reaching movements was $\sim 6 \mathrm{~cm}$. Each target was marked haptically on the touch screen with a cloth disk $(2 \mathrm{~cm}$ diameter $)$, so that subjects received somatosensory feedback on accurate reach trials. Each scan started with a $20 \mathrm{~s}$ rest period in which subjects fixated on a central dot and kept their finger at the central target on the touch screen. This was followed by either a single saccade or a single reach trial that was ignored in the statistical analysis. Eighteen repetitions of each trial type were then presented in a counterbalanced sequence, ending with a $7.5 \mathrm{~s}$ rest period, thus resulting in 440-s-long scans. Subjects participated in three to six scans using their right arm. Before scanning, subjects went through a few practice trials, first outside of the scanner and then inside.

\section{Data analysis}

fMRI data were analyzed with the BrainVoyager QX software package (Brain Innovation, Maastricht, The Netherlands) and with additional in-house software written in Matlab (MathWorks, Natick, MA). Preprocessing of functional scans included discarding the first three to six volumes, intersession and intrasession 3D motion correction, slice scan time correction, and removal of low frequencies up to five cycles per scan (linear trend removal and high-pass filtering). Spatial smoothing was applied to data from the main experiment using a Gaussian filter $(4 \mathrm{~mm}$ full width at half maximum value). The complete dataset was transformed into Talairach space (Talairach and Tournoux, 1988). The cortical surface was reconstructed from the 3D MP-RAGE anatomical images using standard procedures implemented in the BrainVoyager software.

\section{Localizer}

Data from delayed saccade scans were analyzed by computing a crosscorrelation between the signal in each voxel and a sinusoid of a $54 \mathrm{~s}$ period. In areas exhibiting a topographic map, adjacent patches of the cortical gray matter represent adjacent parts of the visual field, and thus successive eye movements to targets around the clock lead to a cortical wave of activity (Sereno et al., 2001; Schluppeck et al., 2005). Data from clockwise and counterclockwise scans were combined by time shifting the time series at each voxel to remove a rough estimate of the hemodynamic delay $(\Delta t=4.5 \mathrm{~s})$ and reversing the counterclockwise scans. All of the scans for each subject were then averaged across sessions, resulting in a time series that reflected the timing of the clockwise scans, in which targets progressed through the right hemifield in the first part of each cycle and through the left hemifield in the second half. This mean time series was used for computing the cross-correlation with the sinusoid. The color coding in Figures 2 and 3 represents the lag yielding the highest correlation, or the phase of the sinusoid, in each voxel. Only clusters of at least six contiguous voxels whose false discovery rate (FDR) was $<0.05$ are shown.

The block alternation scans were also averaged across scans, within each subject. The time course of activity at each voxel was correlated with a binary reference function ( 1 during eye movement blocks and 0 during rest) convolved with a standard hemodynamic response function.

\section{Main experiment}

Statistical analysis of the main experiment was based on linear regression, i.e., a general linear model (Friston et al., 1995). The time course of activity in each patch of cortical gray matter was modeled as a sustained response during each trial, convolved with a standard estimate of the hemodynamic impulse response function (Boynton et al., 1996). This model was constructed for the saccade trials and the reaching trials, with the neutral trials serving as baseline. The model was independently fitted to the activity time course of each voxel, yielding a pair of coefficients, one for saccades and the other for reaches.

\section{Three types of analysis performed using the model}

(1) Contrast analysis. The maps in Figure 3 were obtained by a conjunction of two $t$ tests. Reach-related voxels were defined as those in which the reach coefficient was both positive and significantly greater than the saccade coefficient. Similarly, saccade-related voxels were defined as those in which the saccade coefficient was both positive and significantly greater than the reach coefficient. Only clusters of at least six contiguous voxels whose FDR was $<0.05$ are shown. In frontal areas, we allowed a lower threshold ( $p<0.05$ uncorrected) because we could not localize the putative FEF at the higher threshold; given that FEF has been defined in numerous previous studies, we wanted to ensure that we were not missing any selective activation with an overly conservative statistical threshold.

We also conducted a multisubject contrast analysis using a randomeffect procedure (Friston et al., 1999). In this analysis, separate coefficients were computed for each predictor (saccade and reach) in each subject. A paired $t$ test was then conducted between the saccade and reach coefficients across subjects. Results of the multisubject analysis were projected on an inflated brain from a single subject (see Fig. 6) (supplemental Fig. 1, available at www.jneurosci.org as supplemental material).

(2) Single-condition analysis. The maps in supplemental Figure 2 (available at www.jneurosci.org as supplemental material) highlight voxels in which the coefficient of one predictor or both were significantly larger than zero. The maps were constructed using either a high $(p<$ $10^{-8}$ ) or a low $\left(p<10^{-3}\right)$ per-voxel threshold. Only clusters of at least six contiguous voxels are shown.

(3) Relative-contribution analysis. In supplemental Figure 3 (available at www.jneurosci.org as supplemental material), voxels are color coded according to the relative contribution of the saccade and the reach predictors. First, voxels were selected whose multiple correlation coefficient of the full model $\left(R_{\text {total }}\right)$ was above 0.1 . Then, the model was refitted in each voxel using two reduced models, one excluding the saccade predictor and another excluding the reach predictor. The contribution of each predictor is then computed as the square root of the difference between $R_{\text {total }}$ and the multiple coefficient correlation of the reduced model. The color of each voxel was then determined by the difference between the contributions of the two predictors divided by their sum.

\section{Region of interest analysis}

For 8 of our 10 subjects, phase reversals of the topographic map were used to mark the anterior and posterior borders of areas V7, IPS1, and IPS2 (Schluppeck et al., 2005). The medial and lateral borders of these areas were defined by only including regions on the map that showed a significant response $(\mathrm{FDR}<0.05)$ in both the topography experiment and the saccade block-designed experiment, which presented targets at an eccentricity of $11^{\circ}$. Because the localizer only used this single eccentricity, the regions we defined likely represent only a portion of the true extent of these topographic maps. The maps we obtained in this way were thus a conservative estimate of the extent of these topographically mapped areas.

Regions of interest (ROIs) were also defined in the FEFs, early visual cortex, and primary motor cortex. The saccade topography experiment yielded lateralized activity in the vicinity of the junction between the precentral sulcus (PreCS) and the SFS, which was used to operationally define the FEF, which was situated similarly to previous imaging studies (Corbetta et al., 1998; Grosbras et al., 2005; Hagler and Sereno, 2006). Early visual cortex, mostly corresponding to V1, was defined functionally and anatomically as a region along the calcarine sulcus that exhibited statistically significant activity attributable to the changes in visual stimulation during each trial. To localize motor cortex (mostly corresponding to area M1), we used an "internal-localizer" approach: ROIs were defined using the contrast between reach and saccade trials in half of the main experiment scans, and the other half was used to sample the activity in those now predefined ROIs. Motor cortex was defined based on reach- 
related activity in the dorsal part of the central sulcus (CS) and extending into the precentral gyrus (PreCG). Only subjects who participated in six scans were included in this analysis to maximize statistical power.

Two measures used to quantify the effector selectivity in each ROI (1) Extent of activation. The number of reach- and saccade-related voxels in each ROI. A reach (saccade)-related voxel was defined as one in which the reach (saccade) coefficient was larger than the saccade (reach) coefficient (with no threshold). An "effector selectivity index" was then defined in each ROI as the difference between the number of reach-related voxels and the number of saccade-related voxels, divided by their sum. An ROI that was only active for reach would thus have an index value of 1 ; an ROI that was only active for saccade would have an index of -1 . To calculate the statistical significance of the effector selectivity index, we conducted a bootstrap analysis (Efron and Tibshirani, 1993). First, activation extents for reach and saccade for all subjects in each ROI were pooled together. Numbers were then randomly drawn with repetition from these pooled values and assigned as the reach and saccade extent of activation for each subject. Selectivity index was then recalculated for each subject using the randomly drawn numbers and was averaged across subjects. This was repeated 10,000 times to yield a distribution of average selectivity indices. The values obtained from the original data were compared with the 2.5 percentile of each side of the distribution to determine their significance. To directly compare the selectivity indices between IPS1 and IPS2, we performed an additional bootstrap analysis in which selectivity indices of both areas in each hemisphere were pooled together. Again, values were randomly drawn from this pool and assigned as selectivity indices for IPS1 and IPS2, and the difference between these indices was calculated and averaged across subjects. This was repeated 10,000 times to yield a distribution of difference values, and the 2.5 percentile was again used as the level of significance.

(2) Amplitude of activation. The average signal amplitude in each ROI. Repetitions of each condition, including rest, were first averaged within subject. Next, the averaged time course of activity during the rest periods was subtracted from the averages during the saccade and reach trials. Finally, results were averaged across subjects.

For both quantitative analyses, we were concerned that the imbalance between the number of left and right targets (caused by the omission of a target at the left horizontal meridian; see above, Main experiment) might have caused a difference in the activation patterns between the two hemispheres unrelated to the reach/saccade distinction. We therefore repeated this analysis leaving out all trials in which the targets were at the right horizontal meridian, thus rebalancing the distribution of left and right targets in the analyzed dataset. The results of the two analyses were very similar. Figures 4,5 , and 7 present the results of the balanced analysis, leaving out data from the target placed on the right horizontal meridian.

The results of the amplitude analysis indicated a slightly earlier rise time for reaches compared with saccades. To ensure that this was not a consequence of field- or motion-induced artifacts during the reach trials attributable to the arm movement, we performed the analysis again, splitting the reach trials into "easy" and "hard" trials. Easy trials consisted of movements that only required a rotation of the elbow, and hard trials included extension or retraction of the shoulder joint, movements most likely to compromise the fMRI measurements. All of the effects obtained for the full dataset, including the early rise of reach-related activity, were maintained for each group of movement directions separately.

\section{Results}

To examine the encoding of saccade and reach movements in posterior parietal cortex, we first localized ROIs in eight subjects using topographic mapping as described previously (Sereno et al., 2001; Schluppeck et al., 2005) (see Materials and Methods). Figure 2 presents the results of this mapping in a single subject. The colors represent the fMRI response phases corresponding to angular position in the visual field (indicated by the color wheel). The response in each hemifield was biased for the contralateral visual field: one set of colors (red, magenta, blue) appeared in the

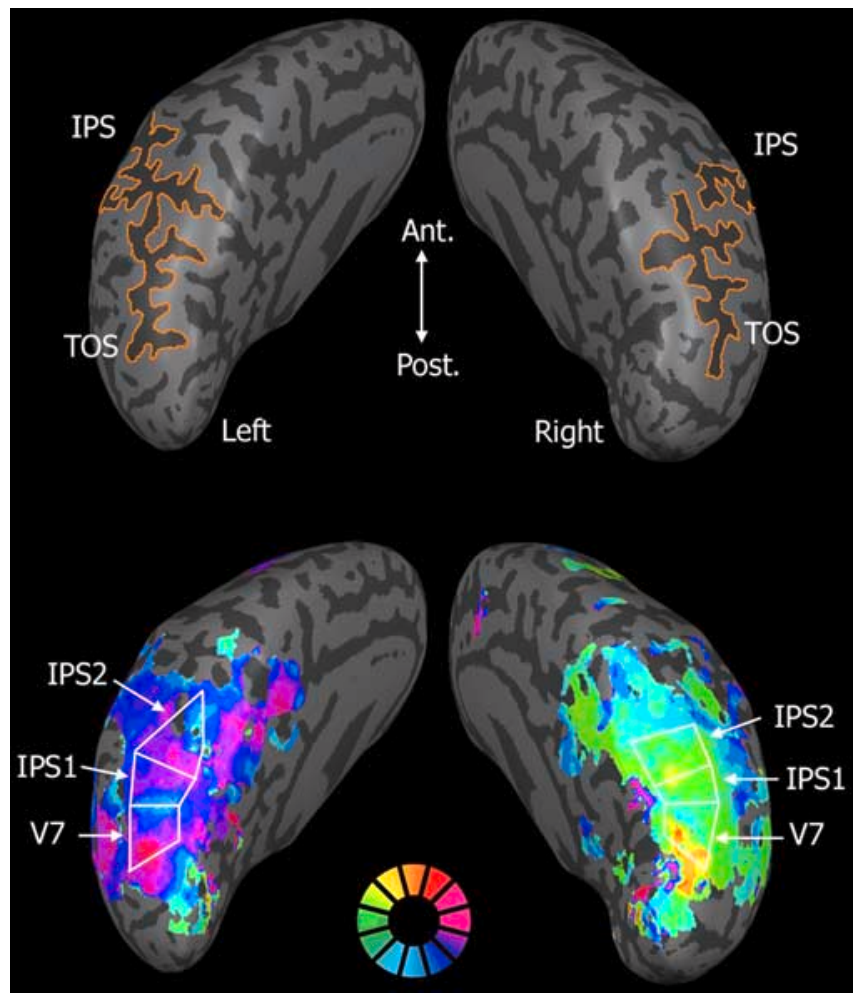

Figure 2. Saccade topography in posterior parietal areas. Results of the localizer experiment in a single representative subject are presented on an inflated brain. The top shows the demarcation of the IPS. The bottom shows the topographic representation of saccade directions in the vicinity of the IPS. Color code denotes saccade direction. Map reversals were used to delineate borders of areas V7, IPS1, and IPS2. Ant, Anterior; Post, posterior.

left hemisphere, whereas a different set of colors (yellow, green, cyan) appeared in the right hemisphere. The phase values progressed smoothly across the transverse occipital sulcus (TOS) and medial aspect of the IPS, implying traveling waves of activity with the periodicity of the targets. At the vicinity of the TOS, the map phase corresponded to a representation of the UVM (red/yellow), smoothly shifting toward a representation of the lower vertical meridian (LVM) (blue/cyan) when moving anteriorly along the IPS. Here the map reversed and shifted smoothly toward a second representation of the UVM and then a second representation of the LVM. Overall, there was an overrepresentation of the horizontal meridian compared with the vertical meridian. This phenomenon has been described previously in high-order visual areas (Larsson and Heeger, 2006) and is most probably a consequence of methodological factors (e.g., blurring inherent in the hemodynamics and post-processing), as well as the asymmetric arrangement of response fields of neurons along the vertical meridian into the ipsilateral hemifield, such that the vertical meridian is represented by neurons with response field centers displaced away from the meridian (Larsson and Heeger, 2006).

The phase reversals of the map were used to define three topographically organized areas: V7, IPS1, and IPS2. Figure 3 presents similar results in three additional subjects. In all hemispheres, at least two phase reversals were observed, thus defining areas V7, IPS1, and IPS2 (Table 1).

Next, we constructed a statistical parametric map of the main experiment in which activity during saccade trials (blue) was contrasted with activity during reach trials (red) (Fig. 3). Superimposing the borders of V7, IPS1, and IPS2 on these maps shows a high degree of intersubject variability but at the same time a 


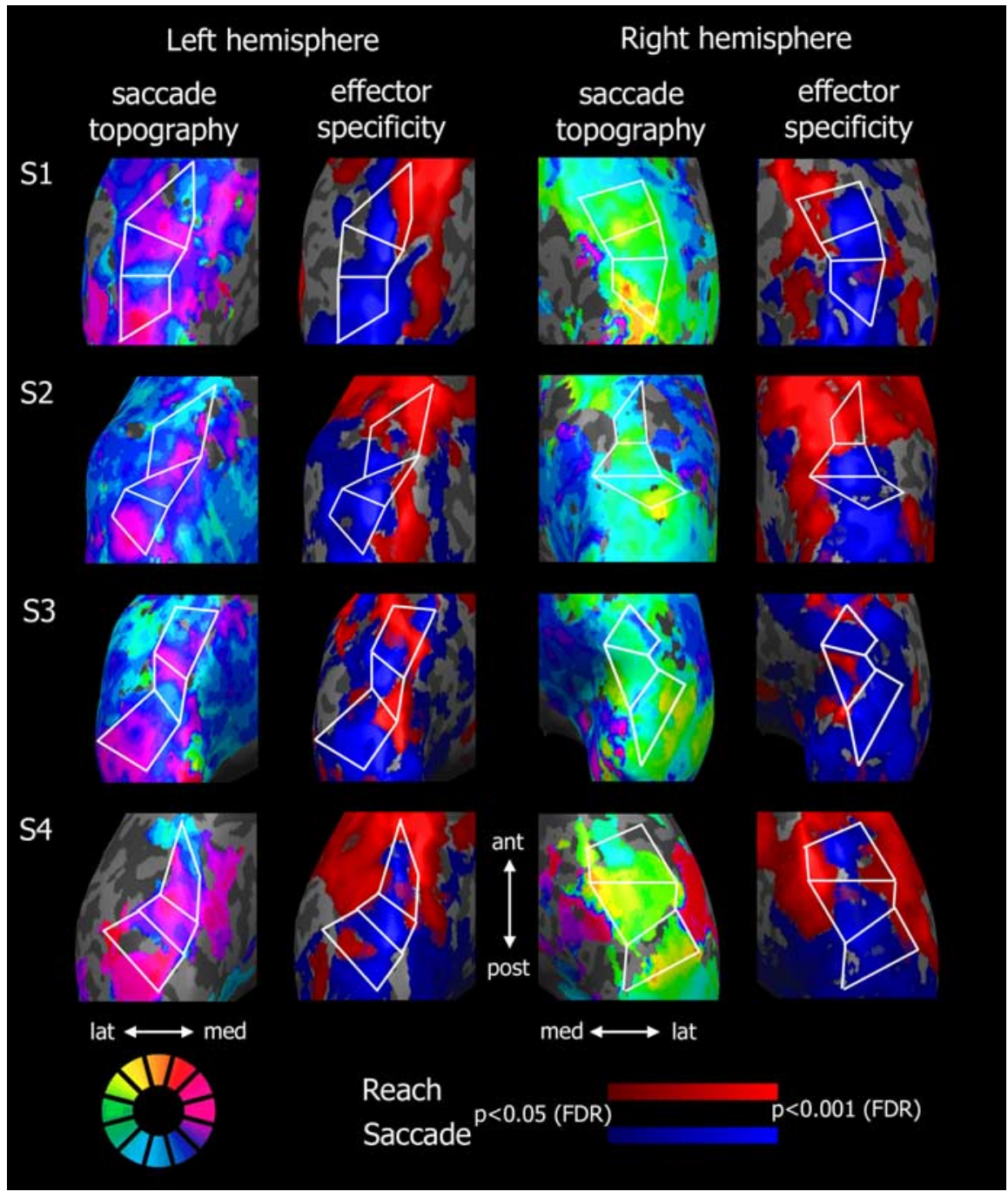

Figure 3. Saccade topography and effector specificity maps in posterior parietal cortex. Results of the localizer experiment (left columns) and the main experiment (right columns) in the subject from Figure 2 and three additional subjects, in the left and right hemispheres. White lines denote the borders of areas V7, IPS1, and IPS2, based on map reversals in the localizer experiment. Red and blue regions showed significantly higher activation to reach and saccade trials, respectively. Only regions whose activation to the preferred effector was positive (above fixation baseline) are shown. ant, Anterior; post, posterior; lat, lateral; med, medial.

sented in Figure $4 B$. Of eight subjects, six had more saccade-related voxels than reach-related voxels in left $\mathrm{V} 7$, and seven showed the same pattern in right V7, whereas all subjects had a higher number of reach-related voxels in left IPS2.

A complementary measure of the preference of each ROI is the average response amplitude across all voxels as a function of effector. Figure 5 presents the time courses sampled from each of the ROIs. Only voxels that exhibited highly significant activation to either saccades or reaching in the main experiment were included in the analysis. Again, in the left hemisphere (Fig. 5, left), there was a significant saccade preference in V7 (two-tailed paired $t$ test of peak activation, $p<0.05$ ), equal activation to saccades and reaching in IPS1 ( $p=$ 0.15 ), and a significant preference for reaches in IPS2 $(p<0.05)$. In the right hemisphere, there was no significant difference of peak amplitude in V7 $(p=0.5)$ and IPS $1(p=0.1)$, but IPS2 exhibited a significant reach preference $(p<0.05)$. Interestingly, rise time for reaches was slightly earlier compared with saccades. Additional analysis showed that this effect was maintained for different movement directions (see Materials and Methods), thus ruling out field- or motion-induced artifacts during the reach trials attributable to the arm movement. Additional research is needed to find out whether the difference in rise time reflects differences in the nature of preparation for arm compared with eye movements.

In a third analysis, we computed statistical parameter maps to assess the preference of each voxel for each effector. Nowhere in parietal cortex did we observe effector specificity, for neither reaches nor saccades. Rather, all areas responded sig-

general transition from saccade preference to reach preference when moving from V7 to IPS2 and also from more lateral to more medial positions within these areas. These general trends are also apparent when the data were combined across subjects, treating intersubject variability as a "random effect" (supplemental Fig. 1, available at www.jneurosci.org as supplemental material).

To quantify the contribution of saccade and reach activity to the total activation in these areas, we performed three complementary analyses. First, we measured the extent of activation by counting the number of saccade- and reach-related voxels in each ROI (see Materials and Methods) (Fig. 4A). In the left hemisphere (Fig. $4 A$, left), V7 contained significantly more saccade than reach voxels ( $p<0.05$, two-tailed paired $t$ test; $n=8$ ), the area defined as IPS1 exhibited a balanced picture with no difference in the number of saccade and reach voxels $(p=0.5)$, and the area defined as IPS2 exhibited a significant bias for reach $(p<$ 0.005). In the right hemisphere, similar trends were observed but did not reach statistical significance (Fig. $4 A$, right). Despite intersubject variability in activation, these biases were generally consistent across subjects, as can be seen in the scatter plots pre- nificantly above baseline to both effectors (supplemental Fig. 2, available at www.jneurosci.org as supplemental material), and both effectors contributed nearly equally to the activity in most areas (supplemental Fig. 3, available at www.jneurosci.org as supplemental material).

Saccade- and reach-related activation was also observed in vast areas outside of posterior parietal cortex. Figure 6 presents an average activation map computed across eight subjects. As expected, somatosensory, motor, and premotor areas responded more strongly to reaching than saccades, whereas visual areas responded (presumably for sensory reasons) more strongly during saccades. In all subjects, a dorsolateral area in the left hemisphere, extending from the CS toward the PreCG, mostly corresponding to M1, was significantly more active for reaching than for saccades (Table 2). In addition, more anterior and more medial areas, corresponding to the premotor cortex (PMC) and the supplementary motor areas (SMA) in both hemispheres, responded more strongly to reaching. Finally, in frontal cortex, stronger saccade-related activity was observed in 8 of 10 subjects in the vicinity of the junction between the PreCS and the medial 
Table 1. Talairach coordinates: parietal areas

\begin{tabular}{|c|c|c|c|c|c|c|c|c|c|c|}
\hline \multirow[b]{2}{*}{ ROI } & \multicolumn{5}{|c|}{ Left } & \multicolumn{5}{|c|}{ Right } \\
\hline & $n$ & $x(\mathrm{~mm})$ & $y(\mathrm{~mm})$ & $z(\mathrm{~mm})$ & Volume $\left(\mathrm{cm}^{3}\right)$ & $n$ & $x(\mathrm{~mm})$ & $y(\mathrm{~mm})$ & $z(\mathrm{~mm})$ & Volume $\left(\mathrm{cm}^{3}\right)$ \\
\hline V7 & 8 & $-26 \pm 2$ & $-81 \pm 5$ & $22 \pm 6$ & $2.8 \pm 1.1$ & 8 & $26 \pm 3$ & $-80 \pm 5$ & $26 \pm 6$ & $2.8 \pm 1.0$ \\
\hline IPS1 & 8 & $-20 \pm 5$ & $-79 \pm 6$ & $34 \pm 6$ & $2.0 \pm 0.5$ & 8 & $22 \pm 2$ & $-77 \pm 7$ & $35 \pm 5$ & $2.3 \pm 0.6$ \\
\hline IPS2 & 8 & $-20 \pm 5$ & $-74 \pm 5$ & $44 \pm 6$ & $2.6 \pm 1.1$ & 8 & $23 \pm 3$ & $-71 \pm 6$ & $44 \pm 4$ & $2.8 \pm 1.2$ \\
\hline
\end{tabular}

Values are mean $\pm S D$

frontal sulcus or medial frontal gyrus (MFG). In a few cases, this activation was more posterior, in the PreCG, or more ventral, in the IFS (Fig. 6, top left; Table 2). Saccade-related activity was also observed in the middle part of the CS in nine subjects in the right hemisphere and in four subjects in the left hemisphere.

To compare the saccade and reaching bias in these areas with the biases observed in posterior parietal cortex, we defined several additional ROIs (bilateral early visual cortex; left motor cortex, because movements were produced with the right hand; and bilateral FEF; see Materials and Methods) and counted the number of saccade- and reach-related voxels in each ROI. V1 exhibited a trend toward saccade preference that did not reach significance in both the left $(390 \pm 50$ vs $190 \pm 90$ $\mathrm{mm}^{3} ; n=8 ; p=0.08$, two-tailed paired $t$ test $)$ and in the right $(340 \pm 60$ vs $170 \pm 60$ $\mathrm{mm}^{3} ; n=8 ; p=0.05$ ) hemispheres. Left motor cortex contained only reachspecific voxels $\left(p<10^{-6} ; n=8\right)$. Finally, FEF showed no significant difference in the number of reach- and saccade-related voxels (left, $p=0.15, n=8$; right, $p=$ $0.17, n=7)$.

Figure 7 presents the effector selectivity indices computed for all of the ROIs in the left (top) and right (bottom) hemispheres, going from occipital through parietal to frontal areas. A gradual shift from saccade preference to reach preference was observed. In the left hemisphere, occipital cortex exhibited a nonsignificant saccade preference. This preference disappeared in IPS1, turned into a significant reach preference in IPS2 ( $p<0.05$, bootstrap analysis; see Materials and Methods), and disappeared again in FEF. Finally, a strong reach preference was observed in the vicinity of left M1. A similar pattern was observed in the right hemisphere, in which a saccade preference was significant in V7 $(p<0.05)$, but the reach preference in IPS2 did not reach significance $(p=$ $0.05)$. A direct test of the difference in effector preference between the transition areas IPS1 and IPS2 did not reach significance in either hemisphere $(p=0.05)$.

\section{Discussion}

\section{Summary}

To test for effector specificity in topographically organized areas of the posterior parietal cortex, we measured cortical activity with fMRI in human subjects while they performed eye and arm movements. We found that these parietal areas showed only slight, albeit significant, preferences for one effector or the other. V7 exhibited stronger responses during saccades than reaches (which might reflect the saccades themselves and/or the visual input induced by the saccades), IPS1 responded equally during saccades and reaches, and IPS2 exhibited stronger responses during reaches than saccades (which might reflect the reaches themselves and/or somatosensory input).

Our results also indicate a reach preference in more medial portions of IPS1 and IPS2 and a saccade preference in more lateral portions of these same areas. This raises the possibility that the human homologs of LIP and PRR divide both IPS1 and IPS2 into medial and lateral subregions. Although this possibility is compatible with the location of LIP and PRR in monkey, the support for it found in our data were weak. This was mainly because our analysis failed to find a consistent geometrical relationship between the IPS1-IPS2 division and this putative medial-lateral division across subjects. To look for such a relationship, we defined the axes of IPS1 and IPS2 in each hemisphere accord- 
Left hemisphere

V7

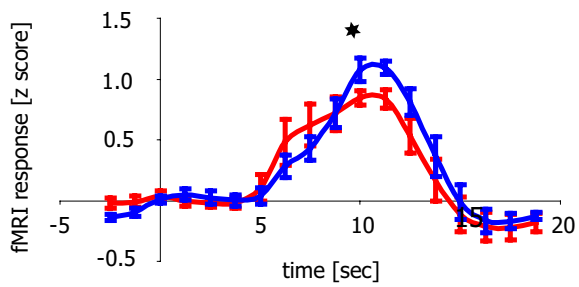

IPS1

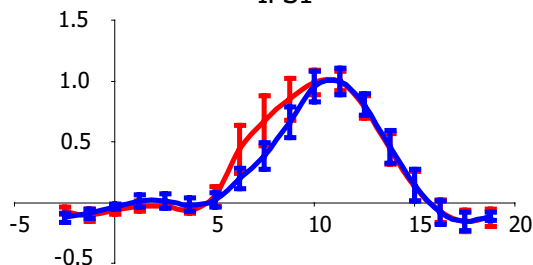

$-0.5$

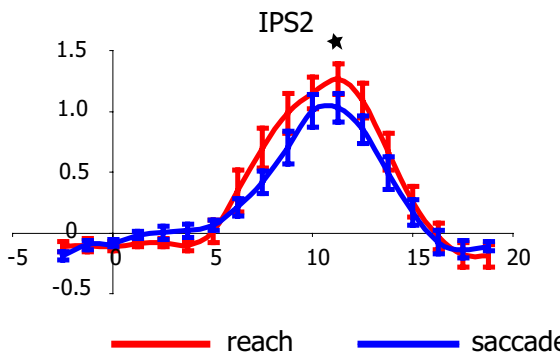

Right hemisphere

V7

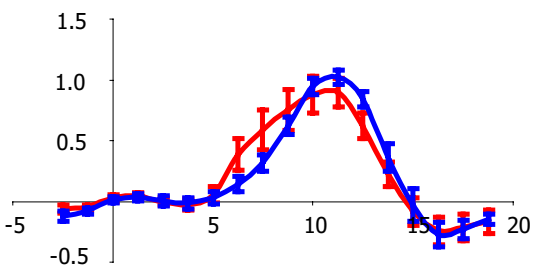

IPS1
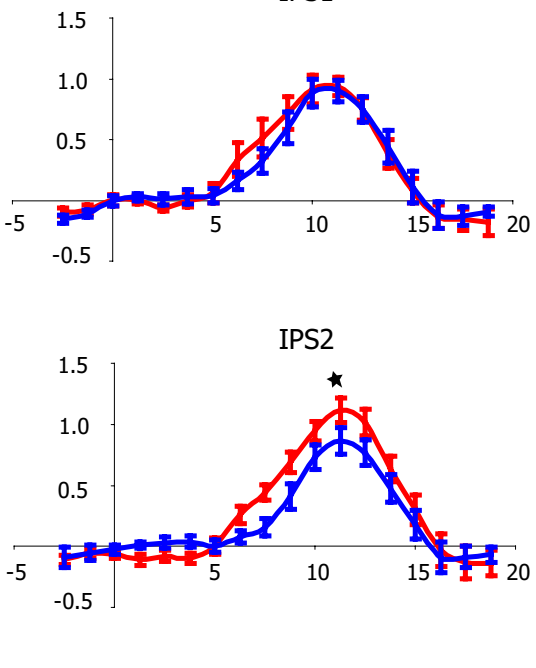

Figure 5. Effector specificity in posterior parietal cortex: amplitude of activation. Response time course for reach (red) and saccade (blue) trials in each ROl averaged across eight subjects and six targets. Activation is aligned with respect to effector cue presentation (time 0). Error bars denote SEM. V7 and IPS2 exhibited slight biases for saccades and reaches, respectively, whereas IPS1 responded equally to saccades and reaches. ${ }^{*} p<0.05$.

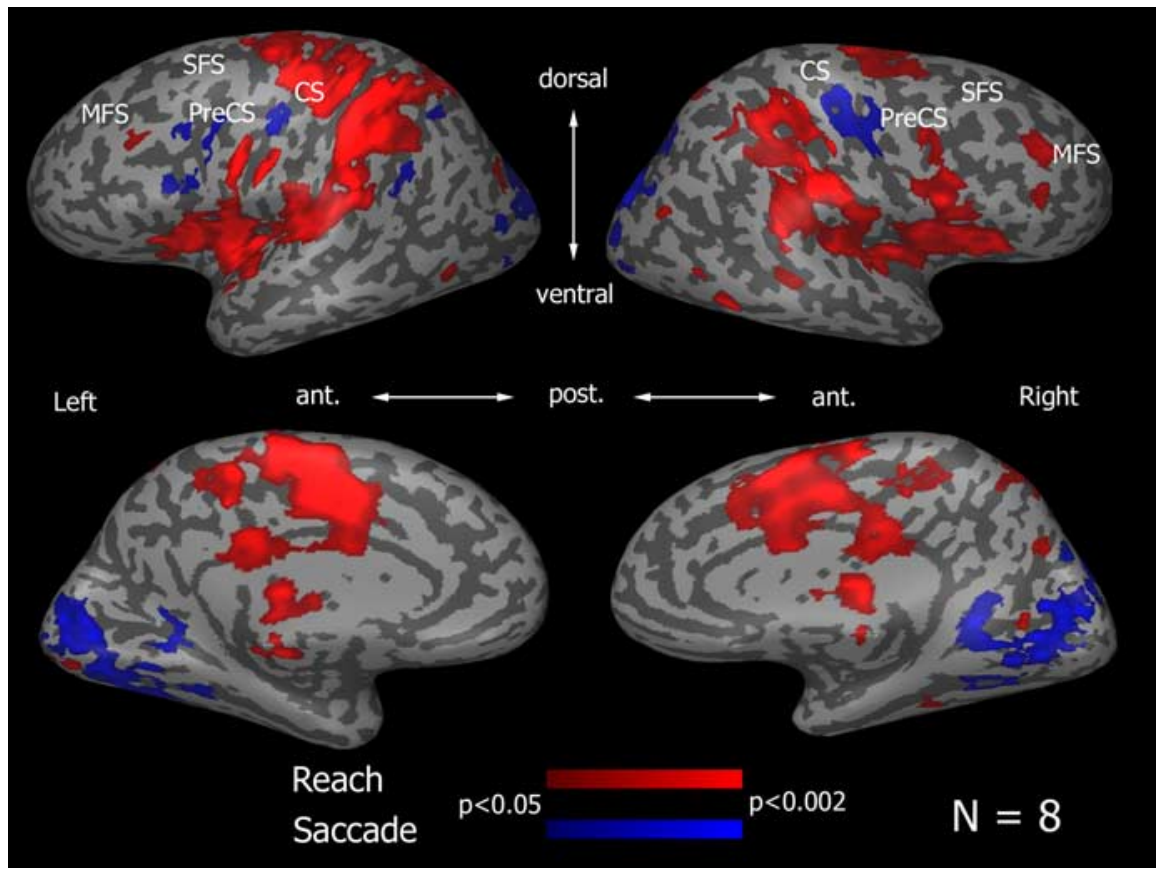

Figure 6. Effector specificity in frontal cortex. Activation map averaged across eight subjects (random effects). Regions in red were significantly more activated to reaching compared with saccades, and regions in blue exhibited the opposite preference. CS, Central sulcus; PreCS, precentral sulcus; MFS, middle frontal sulcus; SFS, superior frontal sulcus; ant, anterior; post, posterior. ing to the direction of the topographic map in each area. We then drew a line connecting the centers of the reach- and saccade-related regions within IPS1 and a similar line within IPS2 and computed the angle between each of these lines and the axis of the corresponding area. Those angles ranged between $70^{\circ}$ and $120^{\circ}$ in different subjects, making a subdivision of IPS 1 and IPS2 into two regions not very likely.

Thus, although our results do generally support the notion of some effector preference in topographically defined parietal areas, they do not appear to support a fully modular effector specificity as has been hypothesized previously. Before drawing that conclusion, however, a number of limitations in the current study must be considered. It should be noted, for example, that reach and saccade trials differed from each other in more than just the motor action performed. First, visual input was slightly different in the two cases. Because the peripheral target remained on for the duration of the trial, in saccade trials this target was brought into fixation after the saccade to the target was made, whereas in reach trials the target remained in the periphery. Second, in reach trials, subjects received somatosensory input from the epithelium of the digit, which was absent in saccade trials. The difference in visual input might explain the saccade preference observed in early visual areas. Differences in activity between saccade and reach trials among the different parietal areas may also be at least partly attributed to differences in visual and somatosensory input. However, if the slight differences in effector preference we observed are only attributable to sensory factors, then the lack of a clear distinction between encoding of different effectors in PPC is even more pronounced than we have suggested here. At the same time, our results do reveal a functional difference between IPS1 and IPS2 in terms of their saccade and reach preferences, which was not reported previously.

\section{Saccade and reach activity in posterior} parietal cortex

Our conclusion that effector preference changes gradually across the cortical surface is highly compatible with previous electrophysiological studies. Different areas in monkey PPC have long been known to exhibit some degree of effector preference (Andersen and Buneo, 2002). Area LIP neurons, for example, respond preferentially to saccades compared with reaches, whereas PRR neurons show the opposite preference both in single-unit 


\begin{tabular}{|c|c|c|c|c|c|c|c|c|c|c|}
\hline \multirow[b]{2}{*}{ ROI } & \multicolumn{5}{|c|}{ Left } & \multicolumn{5}{|c|}{ Right } \\
\hline & $n$ & $x(\mathrm{~mm})$ & $y(\mathrm{~mm})$ & $Z(\mathrm{~mm})$ & Volume $\left(\mathrm{cm}^{3}\right)$ & $n$ & $x(\mathrm{~mm})$ & $y(\mathrm{~mm})$ & $z(\mathrm{~mm})$ & Volume $\left(\mathrm{cm}^{3}\right)$ \\
\hline M1 & 7 & $-33 \pm 6$ & $-26 \pm 3$ & $59 \pm 4$ & $1.2 \pm 0.1$ & & & & & \\
\hline FEF & 8 & $-32 \pm 4$ & $-11 \pm 5$ & $51 \pm 3$ & $1.4 \pm 0.8$ & 8 & $32 \pm 4$ & $-10 \pm 3$ & $51 \pm 3$ & $1.2 \pm 0.2$ \\
\hline Frontal saccade-related area ${ }^{a}$ & 6 & $-41 \pm 3$ & $0 \pm 10$ & $37 \pm 9$ & $1.3 \pm 0.9$ & 4 & $43 \pm 2$ & $-10 \pm 8$ & $45 \pm 6$ & $1.2 \pm 0.9$ \\
\hline
\end{tabular}

Values are mean \pm SD

${ }^{a}$ In some subjects, the frontal saccade-related area corresponded to the dorsolateral prefrontal cortex (Hagler and Sereno, 2006).

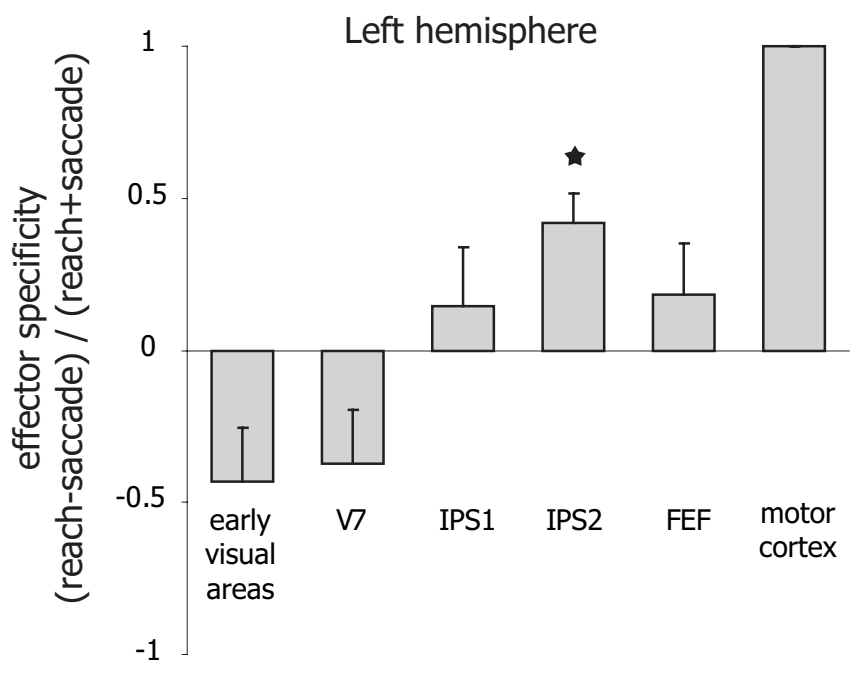

Right hemisphere

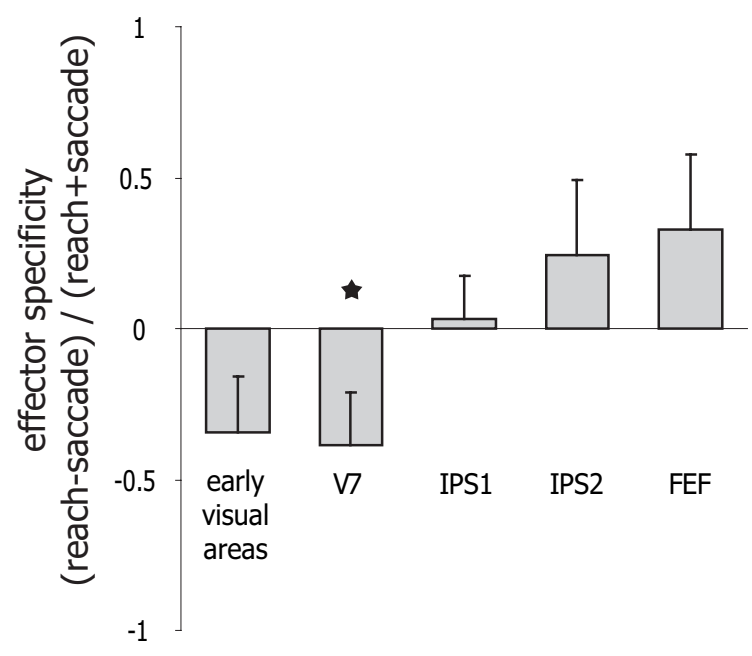

Figure 7. Effector specificity indices in different cortical areas. Saccade and reach bias in each area was computed as the ratio between the difference and the sum of activation extents for each effector. Error bars denote SEM. For left motor cortex, there was no variance between subjects, hence no error bar shown for that point. ${ }^{*} p<0.05$ using a bootstrap method.

studies (Snyder et al., 1997, 1998; Calton et al., 2002) and when the local field potential is examined (Scherberger et al., 2005). However, it has also been widely documented that many neurons in both areas respond to both types of movement (Snyder et al., 1997, 2000b), and some neurons even show a preference for the effector that is nonpreferred by the majority of neurons in each area (Platt and Glimcher, 1997; Snyder et al., 1997). Moreover, Snyder et al. (1997) reported that $32 \%$ of 373 responsive neurons in PPC were significantly active for both saccades and reaches. Even when an association task was used, in which the monkey had to perform arm and eye movements to different targets si- multaneously, $16 \%$ of the neurons remained unspecific. Although the effector-specific neurons were mostly anatomically segregated, $16 \%$ of effector-specific neurons in LIP were arm specific, and $10 \%$ of effector-specific neurons in PRR were saccade specific. That is, not only do reach- and saccade-related neurons coexist in the same cortical areas, but a single neuron may respond equally to both movements.

Recent imaging results using saccade and pointing tasks also support this notion. Essentially every fMRI study conducted to date has reported either areas showing a saccade preference (Simon et al., 2002; Medendorp et al., 2005) or areas showing a pointing preference (Connolly et al., 2000; Astafiev et al., 2003; Connolly et al., 2003) but not a dissociation between two distinct networks with one selective for saccades and the other for reaching (although in some cases this may have been because establishing such a segregation was not the main goal of the paper and in others because the authors only looked for stronger activation for one effector). One positron emission tomography study reported an anatomical segregation for reaching and saccades (Kawashima et al., 1996), but the Talairach coordinates of the reach-related region $(30,-35,50)$ place it in a more anterior area, in the vicinity of primary somatosensory cortex.

Given the substantial intersubject variability observed in our data, these previous results seem highly compatible with what we have observed. On the one hand, the effector preference is so slight that averaging across subjects may be essential to observe it. On the other hand, averaging data across subjects can easily obscure activation that is similar in nature but is located in slightly different regions in different subjects, such that areas preferring a certain effector may be preserved in some studies and not in others.

Interestingly, the general pattern of results we observed in our data were similar in both hemispheres, if weaker in the right hemisphere, although the right arm was used in all the reaching trials. This lack of arm lateralization in PPC is also compatible with previous studies reporting no distinctive effector specificity (Connolly et al., 2003; Medendorp et al., 2005).

Hence, our interpretation of the previously published data are that there is no clear evidence for effector-specific modules in neither monkey LIP and PRR nor human posterior parietal cortex. The gradual transition in effector preference that we observed in IPS1 and IPS2 qualitatively agrees with that observed in monkey LIP and PRR, leaving open the possibility that these areas might be functionally homologous.

\section{Saccade and reach activity in occipital and frontal areas}

Effector-specific modules could, of course, exist in other areas outside of posterior parietal cortex. To begin to test this possibility, we examined effector specificity in a set of additional areas ranging from sensory to motor cortices. We found a gradual and progressive shift from saccade preference to reach preference starting with occipital visual areas and ending with frontal motor areas but no evidence for a distinct boundary along the cortical 
surface at which there is an abrupt transition in effector specificity. Early visual areas, including V1, exhibited a strong saccade preference, which could have resulted from the differences in visual input during reach and saccade trials. Conversely, motor areas M1, PMC, and SMA were exclusively active in reach trials, which could have resulted from the difference in somatosensory input during the reach and saccade trials. However, no other cortical areas responded exclusively for either saccades or reaches. Even the FEF as defined by our topography localizer did not show any significant effector preference, perhaps a surprising finding but one in agreement with previous imaging studies (Connolly et al., 2000; Astafiev et al., 2003; Medendorp et al., 2005) and even a recent electrophysiological report in monkeys (Lawrence and Snyder, 2006). Interestingly, a more ventral area in the vicinity of PreCS and MFG did show a weak saccade preference. This region may correspond to the area identified as FEF by Blanke et al. (2000) using electrical cortical stimulation. In some hemispheres, this area was closer to IFS and may correspond to the dorsolateral prefrontal cortex (Hagler and Sereno, 2006). In either case, this area showed a frontal saccade specificity that exceeded that of the area usually identified as the human FEF. Preferential activity for saccades was also observed in the middle part of the CS, which most likely corresponds to the representation of the face, including the eyelid and the eyeball (Penfield and Jasper, 1954), a region of M1 expected to be active during the saccade trials.

\section{Separability of saccades and reaching: efficient coding constraints}

A correlation between the activity of an area and both saccades and reaches does not imply that that area is causing both types of movements. For example, it is possible that, whenever an arm movement is planned and executed, a plan for an eye movement along the same direction is also formed but not executed. Saccade-related neurons will then be active during reaching movements, although they are not capable of causing such movements. Indeed, activity of this type has been documented (Snyder et al., 1997). Similarly, both saccade and reach planning are correlated with focused spatial attention, and thus both reach areas and saccade areas may be active in either task because attention is reallocated (Silver et al., 2005).

Such reasoning suggests the possibility of a different kind of modular organization in posterior parietal cortex, rooted in the mathematical study of optimal coding. Work in the early visual system has begun to indicate that cortical areas may modularize information in a way that respects the statistical structure of inputs rather than with regard to mathematical convenience (Barlow, 1961; Olshausen and Field, 1996; Simoncelli and Olshausen, 2001). Common visual spatial frequencies, for example, seem to be encoded by more neural elements than uncommon spatial frequencies, despite the fact that a simple Fourier transformation of the visual world would not have this property (Olshausen and Field, 1996). Similarly, it has been suggested that tuning of neurons in motor cortex reflects common aspects of the animal's behavioral repertoire (Aflalo and Graziano, 2006b). Our observations suggest that this may also be true for neural representations of movement in the posterior parietal cortex.

In natural tasks, eye and arm movements are tightly linked. A saccade is usually made to an object or location that will subsequently be the target of a reach (Land et al., 1999; Hayhoe et al., 2003; Hayhoe and Ballard, 2005). Depending on the task, the saccade may follow the reach rather than precede it, but, in most cases, the difference between saccade and reach initiating times is minimal (Hayhoe et al., 2003). Reaching is rarely done without an accompanying saccade, and, although eye movements are sometimes performed without any accompanying limb movement, they are usually made to gather some information that is required for the immediate task (Land et al., 1999).

From the point of view of efficient coding, it therefore makes little sense to have separate machinery for coding similar planned movements that differ only in the effector used to execute them. If the arm and the eyes usually move together toward the same target, a parsimonious coding in motor control areas may well be expected to use a single neuronal population for coding both actions at some levels of the neuraxis. Given the high correlation between the statistics of eye and arm movements, similar activations in brain areas governing the movements of these effectors might well be expected. Interestingly, a recent paper has made a similar suggestion regarding encoding in motor cortex, namely that representations of different effectors are overlapping because the animal uses those effectors together in normal behavior (Aflalo and Graziano, 2006a). At the same time, although arm movements are usually correlated with eye movements, we do occasionally dissociate these effectors. Even monkeys are able to learn to move their eyes toward one target and their arm toward another (Snyder et al., 1997). From this simple observation, one might suggest that, in intermediate levels of the cortical neuraxis, there should be limited but measurable separability of eye- and arm-related neurons. We propose that this is precisely the result that we have observed.

\section{References}

Aflalo TN, Graziano MS (2006a) Possible origins of the complex topographic organization of motor cortex: reduction of a multidimensional space onto a two-dimensional array. J Neurosci 26:6288-6297.

Aflalo TN, Graziano MS (2006b) Partial tuning of motor cortex neurons to final posture in a free-moving paradigm. Proc Natl Acad Sci USA 103:2909-2914.

Andersen RA, Buneo CA (2002) Intentional maps in posterior parietal cortex. Annu Rev Neurosci 25:189-220.

Asanuma C, Andersen RA, Cowan WM (1985) The thalamic relations of the caudal inferior parietal lobule and the lateral prefrontal cortex in monkeys: divergent cortical projections from cell clusters in the medial pulvinar nucleus. J Comp Neurol 241:357-381.

Astafiev SV, Shulman GL, Stanley CM, Snyder AZ, Van Essen DC, Corbetta M (2003) Functional organization of human intraparietal and frontal cortex for attending, looking, and pointing. J Neurosci 23:4689-4699.

Barlow HB (1961) Possible principles underlying the transformations of sensory messages. In: Sensory communication (Rosenblith WA, ed), pp 217-234. Cambridge, MA: MIT.

Blanke O, Spinelli L, Thut G, Michel CM, Perrig S, Landis T, Seeck M (2000) Location of the human frontal eye field as defined by electrical cortical stimulation: anatomical, functional and electrophysiological characteristics. NeuroReport 11:1907-1913.

Blatt GJ, Andersen RA, Stoner GR (1990) Visual receptive field organization and cortico-cortical connections of the lateral intraparietal area (area LIP) in the macaque. J Comp Neurol 299:421-445.

Blatt M, Wiseman S, Domany E (1996) Superparamagnetic clustering of data. Phys Rev Lett 76:3251-3254.

Boynton GA, Engel SA, Glover G, Heeger D (1996) Linear systems analysis of functional magnetic resonance imaging in human V1. J Neurosci 16:4207-4221.

Calton JL, Dickinson AR, Snyder LH (2002) Non-spatial, motor-specific activation in posterior parietal cortex. Nat Neurosci 5:580-588.

Caminiti R, Ferraina S, Johnson PB (1996) The sources of visual information to the primate frontal lobe: a novel role for the superior parietal lobule. Cereb Cortex 6:319-328.

Colby CL, Duhamel JR (1991) Heterogeneity of extrastriate visual areas and multiple parietal areas in the macaque monkey. Neuropsychologia 29:517-537. 
Colby CL, Goldberg ME (1999) Space and attention in parietal cortex. Annu Rev Neurosci 22:319-349.

Connolly JD, Goodale MA, Desouza JF, Menon RS, Vilis T (2000) A comparison of frontoparietal fMRI activation during anti-saccades and antipointing. J Neurophysiol 84:1645-1655.

Connolly JD, Andersen RA, Goodale MA (2003) FMRI evidence for a "parietal reach region" in the human brain. Exp Brain Res 153:140-145.

Corbetta M, Akbudak E, Conturo TE, Snyder AZ, Ollinger JM, Drury HA, Linenweber MR, Petersen SE, Raichle ME, Van Essen DC, Shulman GL (1998) A common network of functional areas for attention and eye movements. Neuron 21:761-773.

Culham JC, Valyear KF (2006) Human parietal cortex in action. Curr Opin Neurobiol 16:205-212.

Efron B, Tibshirani R (1993) An introduction to the bootstrap. London: Chapman and Hall.

Friston J, Homes A, Worsley K, Poline J, Frith C, Frackwowiak R (1995) Statistical parametric maps in functional imaging: a general linear approach. Hum Brain Mapp 2:189-210.

Friston KJ, Holmes AP, Price CJ, Buchel C, Worsley KJ (1999) Multisubject fMRI studies and conjunction analyses. NeuroImage 10:385-396.

Galletti C, Kutz DF, Gamberini M, Breveglieri R, Fattori P (2003) Role of the medial parieto-occipital cortex in the control of reaching and grasping movements. Exp Brain Res 153:158-170.

Glimcher PW (2003) The neurobiology of visual-saccadic decision making. Annu Rev Neurosci 26:133-179.

Gnadt JW, Andersen RA (1988) Memory related motor planning activity in posterior parietal cortex of macaque. Exp Brain Res 70:216-220.

Gottlieb J, Goldberg ME (1999) Activity of neurons in the lateral intraparietal area of the monkey during an antisaccade task. Nat Neurosci 2:906-912.

Grefkes C, Fink GR (2005) The functional organization of the intraparietal sulcus in humans and monkeys. J Anat 207:3-17.

Grosbras MH, Laird AR, Paus T (2005) Cortical regions involved in eye movements, shifts of attention, and gaze perception. Hum Brain Mapp 25:140-154.

Hagler Jr DJ, Sereno MI (2006) Spatial maps in frontal and prefrontal cortex. NeuroImage 29:567-577.

Hayhoe M, Ballard D (2005) Eye movements in natural behavior. Trends Cogn Sci 9:188-194.

Hayhoe MM, Shrivastava A, Mruczek R, Pelz JB (2003) Visual memory and motor planning in a natural task. J Vis 3:49-63.

Kawashima R, Naitoh E, Matsumura M, Itoh H, Ono S, Satoh K, Gotoh R, Koyama M, Inoue K, Yoshioka S, Fukuda H (1996) Topographic representation in human intraparietal sulcus of reaching and saccade. NeuroReport 7:1253-1256.

Land M, Mennie N, Rusted J (1999) The roles of vision and eye movements in the control of activities of daily living. Perception 28:1311-1328.

Larsson J, Heeger DJ (2006) Two retinotopic visual areas in human lateral occipital cortex. J Neurosci 26:13128-13142.

Lawrence BM, Snyder LH (2006) Comparison of effector-specific signals in frontal and parietal cortices. J Neurophysiol 96:1393-1400.

Lewis JW, Van Essen DC (2000) Corticocortical connections of visual, sen- sorimotor, and multimodal processing areas in the parietal lobe of the macaque monkey. J Comp Neurol 428:112-137.

Medendorp WP, Goltz HC, Vilis T, Crawford JD (2003) Gaze-centered updating of visual space in human parietal cortex. J Neurosci 23:6209-6214.

Medendorp WP, Goltz HC, Crawford JD, Vilis T (2005) Integration of target and effector information in human posterior parietal cortex for the planning of action. J Neurophysiol 93:954-962.

Olshausen BA, Field DJ (1996) Natural image statistics and efficient coding $^{\star}$. Network 7:333-339.

Penfield W, Jasper HH (1954) Epilepsy and the functional anatomy of the human brain. Boston: Little Brown.

Platt ML, Glimcher PW (1997) Responses of intraparietal neurons to saccadic targets and visual distractors. J Neurophysiol 78:1574-1589.

Platt ML, Glimcher PW (1998) Response fields of intraparietal neurons quantified with multiple saccadic targets. Exp Brain Res 121:65-75.

Scherberger H, Jarvis MR, Andersen RA (2005) Cortical local field potential encodes movement intentions in the posterior parietal cortex. Neuron 46:347-354.

Schluppeck D, Glimcher P, Heeger DJ (2005) Topographic organization for delayed saccades in human posterior parietal cortex. J Neurophysiol 94:1372-1384.

Schluppeck D, Curtis CE, Glimcher PW, Heeger DJ (2006) Sustained activity in topographic areas of human posterior parietal cortex during memory-guided saccades. J Neurosci 26:5098-5108.

Sereno MI, Pitzalis S, Martinez A (2001) Mapping of contralateral space in retinotopic coordinates by a parietal cortical area in humans. Science 294:1350-1354.

Silver MA, Ress D, Heeger DJ (2005) Topographic maps of visual spatial attention in human parietal cortex. J Neurophysiol 94:1358-1371.

Simon O, Mangin JF, Cohen L, Le Bihan D, Dehaene S (2002) Topographical layout of hand, eye, calculation, and language-related areas in the human parietal lobe. Neuron 33:475-487.

Simoncelli EP, Olshausen BA (2001) Natural image statistics and neural representation. Annu Rev Neurosci 24:1193-1216.

Snyder LH, Batista AP, Andersen RA (1997) Coding of intention in the posterior parietal cortex. Nature 386:167-170.

Snyder LH, Batista AP, Andersen RA (1998) Change in motor plan, without a change in the spatial locus of attention, modulates activity in posterior parietal cortex. J Neurophysiol 79:2814-2819.

Snyder LH, Batista AP, Andersen RA (2000a) Intention-related activity in the posterior parietal cortex: a review. Vision Res 40:1433-1441.

Snyder LH, Batista AP, Andersen RA (2000b) Saccade-related activity in the parietal reach region. J Neurophysiol 83:1099-1102.

Talairach J, Tournoux P (1988) Co-planar stereotaxic atlas of the human brain. New York: Thieme Medical Publishers.

Thier P, Andersen RA (1996) Electrical microstimulation suggests two different forms of representation of head-centered space in the intraparietal sulcus of rhesus monkeys. Proc Natl Acad Sci USA 93:4962-4967.

Thier P, Andersen RA (1998) Electrical microstimulation distinguishes distinct saccade-related areas in the posterior parietal cortex. J Neurophysiol 80:1713-1735. 